\title{
Two Methods for the Computation of Commercial Pipe Friction Factors
}

\author{
D. H. Yoo and V. P. Singh, F.ASCE ${ }^{2}$
}

\begin{abstract}
Two methods are proposed for the computation of friction factors of commercial pipes. The first method applies the mean value of the zero velocity point (MZVP) to a theoretical friction factor equation, and the other directly computes the mean friction factor (MFF) by averaging the friction factor of both the smooth and rough walls while considering their relative contribution. The MFF method is preferred, because it is simple but covers all the flow characteristics of commercial pipes. Both MFF and MZVP methods consider two parts of a wall with different roughness heights: One part is rough and the other is smooth. A regression analysis was performed to determine optimum values of the roughness height and probability of encountering each part, using several sets of field data, including galvanized iron, wrought iron, cast iron, concrete, riveted steel, and concrete. The analysis showed that both the roughness height and the relative contribution of the rough part are strongly dependent on the pipe diameter. The MFF method gave an average error of less than $3 \%$, whereas the traditional Colebrook-White equation gave an average error of more than $11 \%$ when compared with Colebrook's data.
\end{abstract}

DOI: 10.1061/(ASCE)0733-9429(2005)131:8(694)

CE Database subject headings: Pipe flow; Friction; Roughness; Velocity; Computation; Flow characteristics.

\section{Introduction}

Flow in hydraulic conduits is always subject to resistance and energy dissipation. Hydraulic resistance, often expressed as a coefficient or friction factor, is the basic information needed in hydraulic computation and design. Since the pioneering contributions by Weisbach (1845), Darcy (1857), Boussinesq (1877), and Reynolds (1895) among others, the hydraulics of flow resistance has been a subject of much interest and investigation. Many flow resistance equations have been reported for pipes as well as channels in the hydraulic literature. A comprehensive discussion of flow resistance equations was presented by Singh (1996), and Yen (2002) provided a historical perspective for open channel flow resistance.

An early contribution is the Darcy-Weisbach equation expressed as

$$
h_{f}=f \frac{L}{D} \frac{V^{2}}{2 g}
$$

where $h_{f}=$ frictional head loss; $f=$ Darcy-Weisbach friction factor; $L=$ pipe length; $D=$ pipe diameter; $V=$ cross-sectional mean velocity; and $g=$ acceleration due to gravity. Based on the data collected primarily for circular-type pressure conduits and for

\footnotetext{
${ }^{1}$ Professor, Dept. of Civil Engineering, Ajou Univ., Suwon 442-749, Korea.

${ }^{2}$ Arthur K. Barton Professor, Dept. of Civil and Environmental Engineering, Louisiana State Univ., Baton Rouge, LA 70803-6405 (corresponding author). E-mail: cesing@1su.edu

Note. Discussion open until January 1, 2006. Separate discussions must be submitted for individual papers. To extend the closing date by one month, a written request must be filed with the ASCE Managing Editor. The manuscript for this paper was submitted for review and possible publication on October 29, 2002; approved on December 21, 2004. This paper is part of the Journal of Hydraulic Engineering, Vol. 131, No. 8, August 1, 2005. CASCE, ISSN 0733-9429/2005/8-694-704/ $\$ 25.00$.
}

open channels, Williams and Hazen (1933) proposed an empirical equation for estimating the mean velocity of flow in pipes. The empirical equation of Hazen-Williams in SI units is given by

$$
V=0.85 C_{\mathrm{HW}} R^{0.63} S^{0.54}
$$

where $C_{\mathrm{HW}}=$ Hazen-Williams coefficient; $R=$ hydraulic radius; and $S=$ pipe slope.

When using the Hazen-Williams equation for commercial pipes, a multitude of uncertainties are encountered. For example, the constant used in the Hazen-Williams equation is known to vary significantly, depending on the diameter and the type of pipe. Swamee and Jain (1976) developed several equations relating the Hazen-Williams parameter to the pipe diameter. Kamand (1988) also argued that the Hazen-Williams equation is not appropriate in the case of low Reynolds numbers and/or small-diameter pipes, and introduced a correction factor to achieve a better agreement with the Colebrook-White equation.

Using the data collected by various investigators and employing Prandtl's mixing length theory Prandtl (1925), Colebrook and White (1937), and Colebrook (1938) developed a semitheoretical equation for friction factors of commercial pipes. The ColebrookWhite equation is represented by:

$$
\frac{1}{\sqrt{f}}=-2 \log \left(\frac{2.5}{\mathrm{R} \sqrt{f}}+\frac{\kappa_{w}}{3.7 D}\right)
$$

where $\mathrm{R}=$ Reynolds number and $\boldsymbol{\kappa}_{w}=$ roughness height. Colebrook found that the roughness heights varied significantly but that they were randomly scattered against the pipe diameter. Thus, he proposed using an average value of the roughness height for each type of commercial pipe, with some error tolerated. Moody (1944) presented a diagram of commercial pipe friction factors based on the Colebrook-White equation, which has been extensively used for practical applications.

The U.S. Bureau of Reclamation (1965) reported large amounts of field data (more than 25 sets) on commercial pipes: concrete, continuous-interior, girth-riveted, and full-riveted steel 
pipes. Due to large variations in the field data, average friction factors were used for simplicity. The researchers of the Bureau of Reclamation found that some of the field data collected could not be explained by the Colebrook-White equation, since the variation of the data followed the curve of transitional turbulent flow which is omitted in the composition of the Colebrook-White equation. The Bureau of Reclamation report (1965) asserted that the Colebrook-White equation was found inadequate over a wide range of flow conditions.

Moreover, several researchers have found that the ColebrookWhite equation is inadequate for pipes smaller than $2.5 \mathrm{~mm}$. Wesseling and Homma (1967) suggested using a Blasius-type equation or a power law with minor modifications instead of the Colebrook-White equation. They recommended using larger values of the proportionality factor for smaller-size pipes. von Bernuth and Wilson (1989) conducted laboratory experiments and attempted to find the optimum value of the roughness height of PVC pipes for the Colebrook-White equation and then the value of the friction factor of PVC pipes. Their computation results were, however, quite different from those obtained in the laboratory when using the Colebrook-White equation. Instead they proposed to employ a Blasius-type equation with minor modifications. The friction factor determined from laboratory data decreases with an increase in the Reynolds number even after a certain critical value, whereas the friction factor of the Colebrook-White equation tends to be constant with an increase in the Reynolds number. In recent years Jain et al. (1978), Kamand (1988), and Bagarello et al. (1995) discouraged the use of the Colebrook-White equation for the computation of commercial pipe friction factors, particularly for small-diameter pipes. Instead they suggested using a power law with minor modifications.

This study proposes two new methods for estimating the friction factor of commercial pipes: one combining the zero velocity points and the other combining the friction factors of the smooth and rough parts of the pipe. Using the data reported by Colebrook (1938), the U.S. Bureau of Reclamation (1965) and von Bernuth and Wilson (1989), a regression analysis was performed to determine the optimum values of the roughness height and the probability of occurrence of both parts. The roughness height and probability of occurrence of the rough part were then related to the pipe diameter, while the roughness height of the smooth part was determined for various pipe materials.

\section{Determination of Friction Factor}

Prandtl's mixing length theory describes the near-wall turbulent flow characteristics reasonably well, where the velocity distribution is expressed as

$$
\frac{u}{u_{*}}=\frac{1}{\kappa} \ln \frac{z}{z_{0}}
$$

where $u=$ local flow velocity; $u_{*}=$ frictional velocity given by $\sqrt{\tau / \rho} ; \tau=$ wall shear stress; $\rho=$ water density; $z=$ height above the wall; $\kappa=$ von Karman constant $(=0.4)$; and $z_{0}=$ zero velocity point at which the local velocity vanishes according to the logarithmic law. Assuming that the velocity is symmetric about the pipe centerline and the shear stress is constant along the perimeter

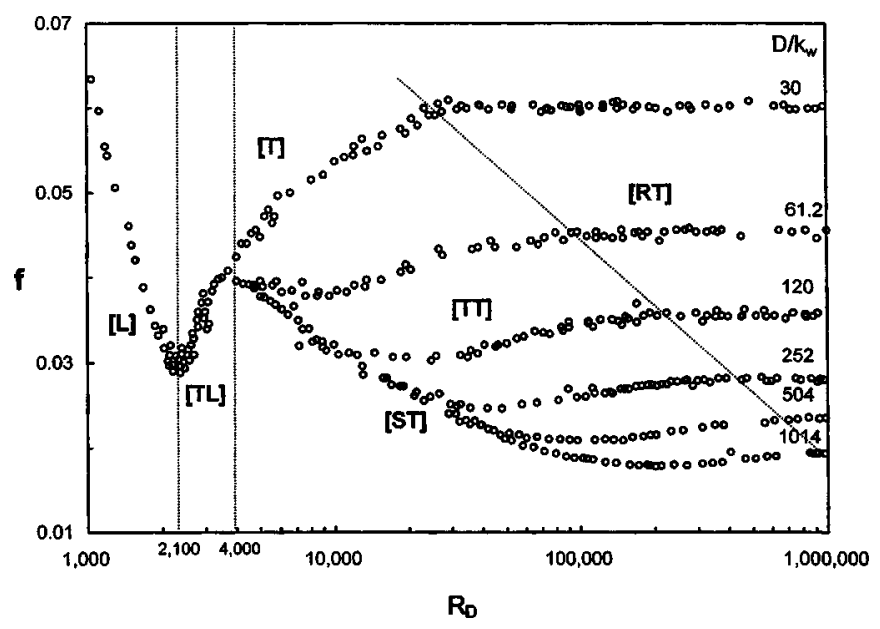

Fig. 1. Darcy-Weisbach friction factor of uniformly rough circular pipe flow [Laboratory results from Nikuradse (1933)]: L=laminar; $\mathrm{RT}=$ rough turbulent flow; $\mathrm{ST}=$ smooth turbulent flow; $\mathrm{T}=$ turbulent flow; $\mathrm{TL}=\mathrm{LR}=$ transitional flow between laminar and rough turbulent flow; and $\mathrm{TT}=$ transitional turbulent flow

of a circular wall, integration of Eq. (4) yields the cross-sectional mean velocity, $V$, as:

$$
V=\frac{Q}{A}=\frac{1}{\pi r^{2}} \int_{0}^{r} 2 \pi(r-z) \frac{u_{*}}{\kappa} \ln \frac{z}{z_{0}} d z
$$

where $r=$ radius of the pipe; $Q=$ discharge; and $A=$ cross-section area. After integration this becomes

$$
\frac{1}{\sqrt{C}}=\frac{1}{\kappa}\left[\ln \frac{r}{z_{0}}-1.5\right]
$$

where the friction factor $C$ is defined by

$$
u_{*}=\sqrt{C} V \text { or } \tau=\rho C V^{2}
$$

According to laboratory results by Nikuradse (1933) as shown in Fig. 1, the Darcy-Weisbach friction factor, $f$, is given as

$$
\begin{array}{ll}
\frac{1}{\sqrt{f}}=2 \log \mathrm{R} \sqrt{f}-0.8 & \text { for }[\mathrm{ST}] \\
\frac{1}{\sqrt{f}}=2 \log D_{\mathrm{\kappa}}+1.14 & \text { for }[\mathrm{RT}]
\end{array}
$$

where $f=8 C ; \mathrm{R}=$ Reynolds number given by $V D / \nu ; D=$ pipe diameter; $\nu=$ kinetic viscosity; $D_{\kappa}=D / \kappa_{w} ; \kappa_{w}=$ equivalent roughness height; $[\mathrm{ST}]$ indicates the smooth turbulent flow or turbulent flow in a hydraulically smooth pipe; and [RT] the rough turbulent flow or turbulent flow in a hydraulically rough pipe.

Expressing $C$ in terms of $f$ in Eq. (6) and using the common logarithm instead of natural logarithm, one obtains

$$
\frac{1}{\sqrt{f}}=2 \log \frac{D}{z_{0}}-1.94
$$

Matching Eq. (8) with Eq. (10) and Eq. (9) with Eq. (10), respectively, yields the zero velocity point as:

$$
\begin{array}{ll}
z_{0 S T}=0.10 \frac{v}{u_{*}} & \text { for }[\mathrm{ST}] \\
z_{0 R T}=0.03 \kappa_{w} & \text { for }[\mathrm{RT}]
\end{array}
$$


There is a significant portion existing between smooth turbulent flow and rough turbulent flow, as shown in Fig. 1, which is normally called transitional turbulent flow, and it may also occur in flows in commercial pipes. This region is herein called the transitional turbulent flow [TT]. Using regression analysis, a simple equation was developed for this region:

$$
f=f_{R T}-f_{A} \cos \left(0.8 \ln R_{\kappa}-4.06\right) \text { for }[\mathrm{TT}]
$$

where $f_{R T}=f$ at rough turbulent flow; $f_{A}=0.06 D_{\kappa}^{-0.5}$; and $\mathrm{R}_{\mathrm{K}}=V \mathrm{\kappa}_{w} / \nu$. The boundary condition for [TT] was determined by $3<\mathrm{R}_{*_{\mathrm{\kappa}}}<100$ or $81.5<\mathrm{R}_{\mathrm{\kappa}}<1140$ where $\mathrm{R}_{*_{\mathrm{K}}}=u_{*} \kappa_{w} / v$. For circular pipes the second condition for using $R_{\kappa}$ can also be satisfactorily employed to determine the flow condition, and it is easier to use than the first condition as $u_{*}$ can be estimated implicitly through an iterative process.

When the roughness ratio $D_{\mathrm{K}}$ is less than 50, the flow condition moves directly from laminar to rough turbulent flow bypassing the smooth turbulent and transitional turbulent flow regions. In this case the friction factor is determined by regression as

$$
f=0.03+\left(f_{R T}-0.03\right) \tanh \left[\frac{\lambda(\ln \mathrm{R}-7.64)}{\ln D_{\mathrm{K}}-0.61}\right] \text { for }[\mathrm{LR}]
$$

where $\lambda=3.5-0.05 D_{\mathrm{K}}$ and [LR] indicates the transitional flow between laminar and rough turbulent flows.

Colebrook and White (1937) developed an equation for determining the friction factor for commercial pipes by combining Eqs. (11) and (12) to represent the zero velocity point of the commercial pipe and then to substitute the combined function into Eq. (6). They suggested the zero-velocity point representing a commercial pipe as

$$
z_{0 C}=z_{0 S}+z_{0 R}
$$

where subscripts $C, S$, and $R$ represent "commercial pipe," "smooth pipe," and "rough pipe," respectively. They assumed that the smooth pipe is hydraulically smooth or the flow in the smooth part is at the flow condition [ST] and that the rough pipe is hydraulically rough or the flow in the rough part is at the flow condition [RT]. This may not be valid when the pipe size is very small and the velocity is very high, since the flow in the smooth part can also be transitional turbulent or even rough turbulent. Replacing the zero velocity point of Eq. (6) with Eq. (15), Colebrook (1938) derived the friction factor equation (3) for commercial pipes.

Colebrook (1938) analyzed four sets of field data of commercial pipes observed by various researchers: galvanized iron, wrought iron, asphalted cast iron, and uncoated cast iron pipes. He presented three figures displaying roughness height versus pipe diameter, which show random variations of roughness heights for all types of commercial pipes: $0.025-0.27 \mathrm{~mm}$ for galvanized iron pipes, $0.03-0.25 \mathrm{~mm}$ for asphalted cast iron pipes, and $0.01-0.1 \mathrm{~mm}$ for wrought iron pipes as shown in Fig. 2. The figures do not show any dependency of roughness height on the pipe diameter. It is suspected that this is the reason why he chose the mean value of roughness height for each type of pipe. But it is clear that the application of the mean value of the roughness height, due to its large variation, would often result in errors of more than $10 \%$.
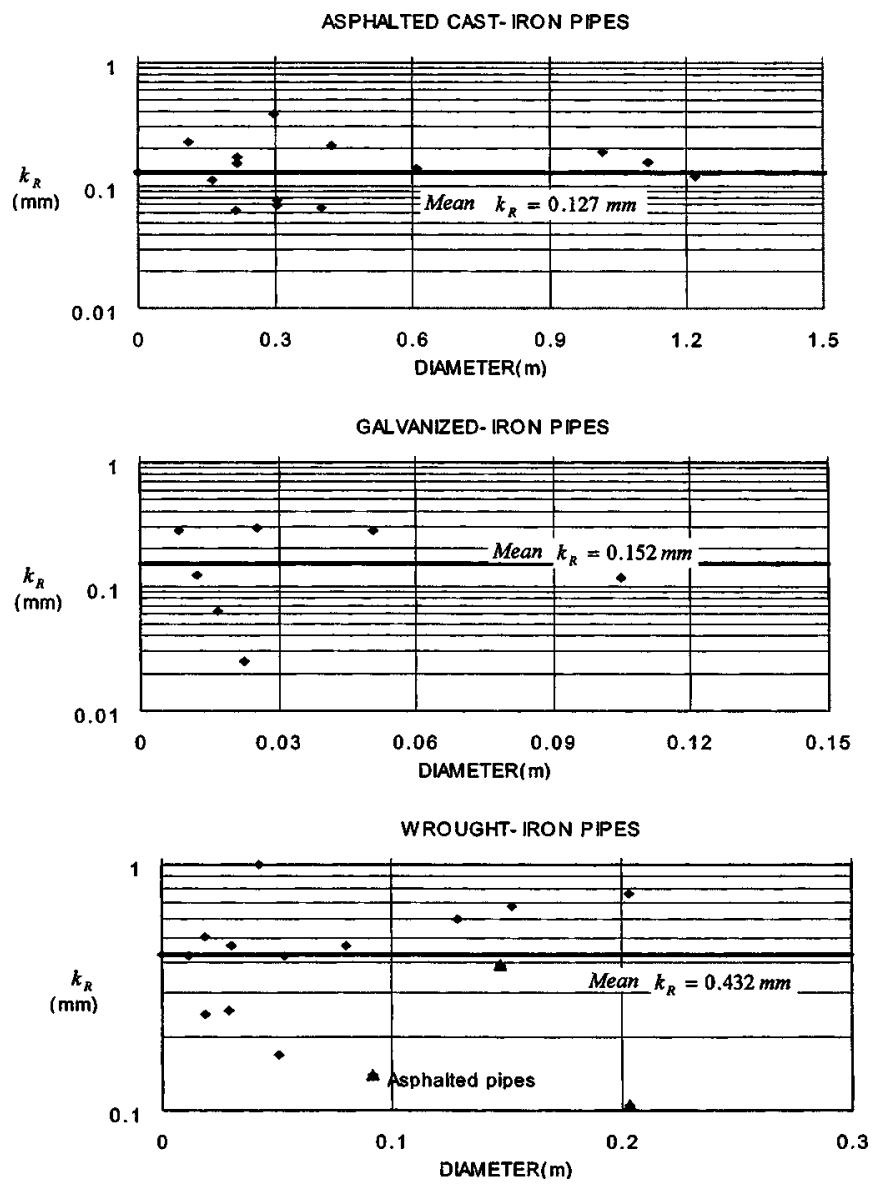

Fig. 2. Variation of roughness height against pipe diameter with Colebrook-White equation after Colebrook (1938). The solid line corresponds to the mean roughness height.

\section{Suggested Modifications for the Colebrook-White Equation}

The Colebrook-White equation can be improved by eliminating its deficiencies.

1. The simple summation of the zero velocity point as done in Eq. (15) is inaccurate with respect to the influence by each wall. The summation expressed by Eq. (15) implies that each wall (smooth and rough) occupies the pipe $100 \%$, which means that the same space is occupied $200 \%$. The influence of each wall should be considered rather than simply assuming an equal influence (100\%) for each wall. This influence can also be interpreted as a probability. Colebrook (1938) even assumed that both the smooth and rough walls dominate a single pipe.

2. The flow in the smooth part of a commercial pipe may not always be hydraulically smooth or smooth turbulent, and the flow in the rough part of a commercial pipe may not always be hydraulically rough or rough turbulent. The flow in the smooth part of a commercial pipe can be one of these flow conditions, such as laminar, transitional laminar, smooth turbulent, transitional turbulent or rough turbulent, although the flow is likely to be smooth turbulent.

3. The smooth part is not perfectly smooth but it has a definite roughness height, although it may be small. Therefore, the flow in the smooth part can be rough turbulent or transitional 
Welding 0

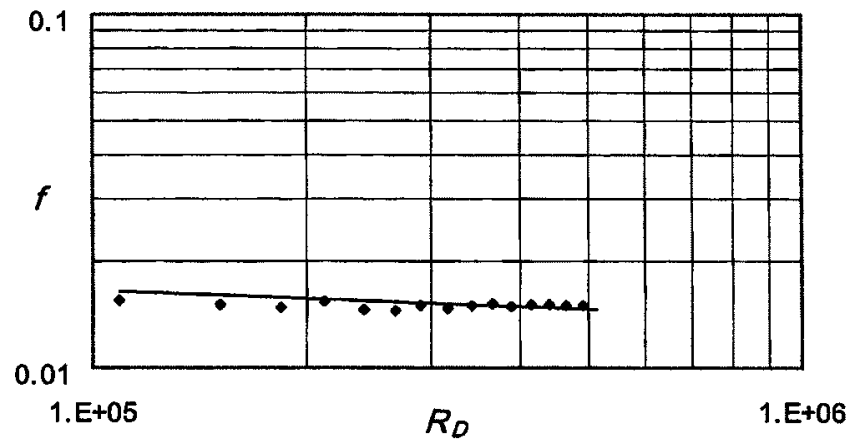

(a)

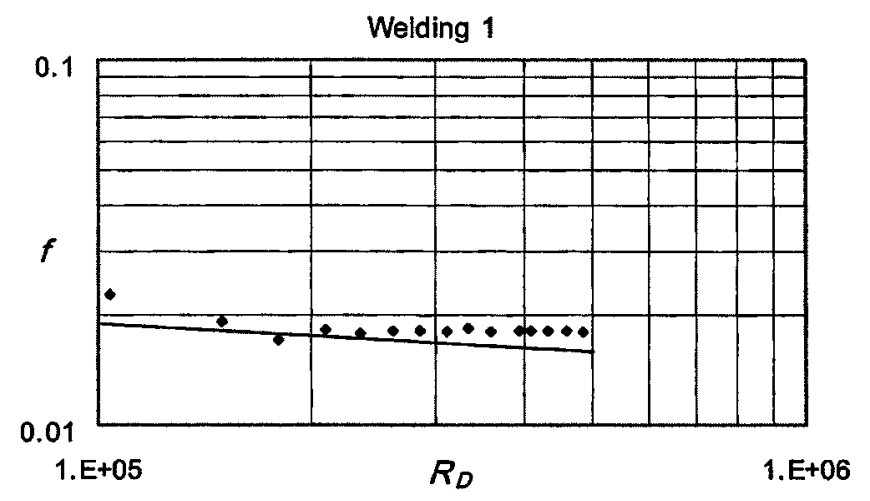

(b)

Fig. 3. Variation of friction factor of galvanized iron pipe. The solid dots indicate the measured data while the solid line indicates the values given by the Colebrook-White equation. (a) No-connection part; and (b) one connection part.

turbulent when the flow has a very high velocity or more importantly the pipe diameter is small.

4. The transitional turbulent flow [TT] and transitional flow [T] may occur quite often for both parts, and hence its omission in the formulation of the Colebrook-White equation may result in error (see the section entitled "Roughness Height and its Influence").

In order to mitigate the deficiencies, two methods are proposed for computation of commercial pipe friction factors. The first method is similar to the method of Colebrook and White, but the mean zero velocity point is computed by considering the percentage of each representative part. Then the mean zero velocity point is substituted into Eq. (6) to estimate the commercial pipe friction factor. This is designated as the method of mean zero velocity point $[\mathrm{MZVP}]$. The second method is that the mean friction factor is directly computed by averaging the values of two representative parts, and is designated as the method of mean friction factor [MFF].

\section{Method of Mean Zero Velocity Point}

Using the MZVP, the zero velocity point of commercial pipes is computed as

$$
z_{0 C}=P_{S} z_{0 S}+P_{R} z_{0 R}
$$

where $P_{S}=$ probability (or percentage) of a smooth wall; $P_{R}=$ probability (or percentage) of a rough wall; and $z_{0 S}$ is not necessarily the same as $z_{0 S T}$. Similarly, $z_{0 R}$ is not necessarily the same as $z_{0 R T}$. Further, $P_{S}+P_{R}=1$. Eq. (15), which is the basis of the Colebrook-White equation, shows that $P_{S}+P_{R}=2$, which is clearly incorrect. If it is assumed that $z_{0 S}=z_{0 S T}=0.1 v / u_{*}$ and $z_{0 R}$ $=z_{0 R T}=0.03 \kappa_{w}$ and Eq. (16) is substituted into Eq. (6), one obtains:

$$
\frac{1}{\sqrt{f}}=-2 \log \left(\frac{2.5 P_{S}}{\mathrm{R} \sqrt{f}}+\frac{P_{R} \boldsymbol{\kappa}_{w}}{3.7 D}\right)
$$

The assumption that $P_{S}=1$ and $P_{R}=1$ in Eq. (17), which is certainly not true, leads to the Colebrook-White equation [Eq. (3)]. Using the explicit form of the zero velocity point for smooth turbulent flow, $z_{0 S T}=0.58 D \mathrm{R}^{-0.89}$ after Barr (1976), $z_{0 R T}=0.03 \kappa_{w}$ and when $z_{0 S}=z_{0 S T}$ and $z_{0 R}=z_{0 R T}$, the friction factor for commercial pipes is given as:

$$
\frac{1}{\sqrt{f}}=-2 \log \left(\frac{5.13 P_{S}}{\mathrm{R}^{0.89}}+\frac{P_{R} \kappa_{w}}{3.7 D}\right)
$$

\section{Method of Mean Friction Factor}

Using the MFF method, the friction factor of commercial pipes is computed as

$$
f_{C}=P_{S} f_{S}+P_{R} f_{R}
$$

where $f_{C}=$ mean friction factor of commercial pipes; $f_{S}=$ friction factor of the smooth part; and $f_{R}=$ friction factor of the rough part. As for the zero velocity point, $f_{S}$ is not necessarily the same as $f_{S T}$, and $f_{R}$ is not necessarily the same as $f_{R T} . f_{S}$ or $f_{R}$ can be one of the friction factors: $f_{L}, f_{L R}, f_{S T}, f_{T T}, f_{R T}$ or $f_{L R}$, depending on the flow condition of each part with different roughness heights.

Although the MZVP method or the Colebrook-White approach is expressed by one single equation, the mean value of the zero velocity point is considered to be incorrect. If the roughness is irregular but evenly distributed over the whole pipe, the roughness height should be represented by one single average value or a representative value rather than two values which result from the combination of two equations. Furthermore, the flow in commercial pipes can be laminar, which cannot be described by the concept of zero velocity point. It is also difficult to describe the transitional flow between laminar and rough turbulent flow using the concept of zero velocity point, which may occur quite often in small-size pipes. Therefore, MFF is considered to be more appropriate than MZVP for the estimation of friction factors of commercial pipes in field situations.

\section{Roughness Height and its Influence}

Assuming the rough part of a commercial pipe has the same proportion as the smooth part, Colebrook (1938) computed the roughness heights of various commercial pipes and found a wide variation of roughness height with the pipe diameter. As shown in Fig. 2, when using the Colebrook-White equation, the roughness height of the galvanized iron pipe varies from 0.025 to $0.27 \mathrm{~mm}$ with no particular dependency on the pipe diameter. He also obtained similar results for other types of pipes.

In the present analysis, the MFF method was applied to compute the roughness heights and the percentages of the rough part of commercial pipes using the least squares method. From a set of 

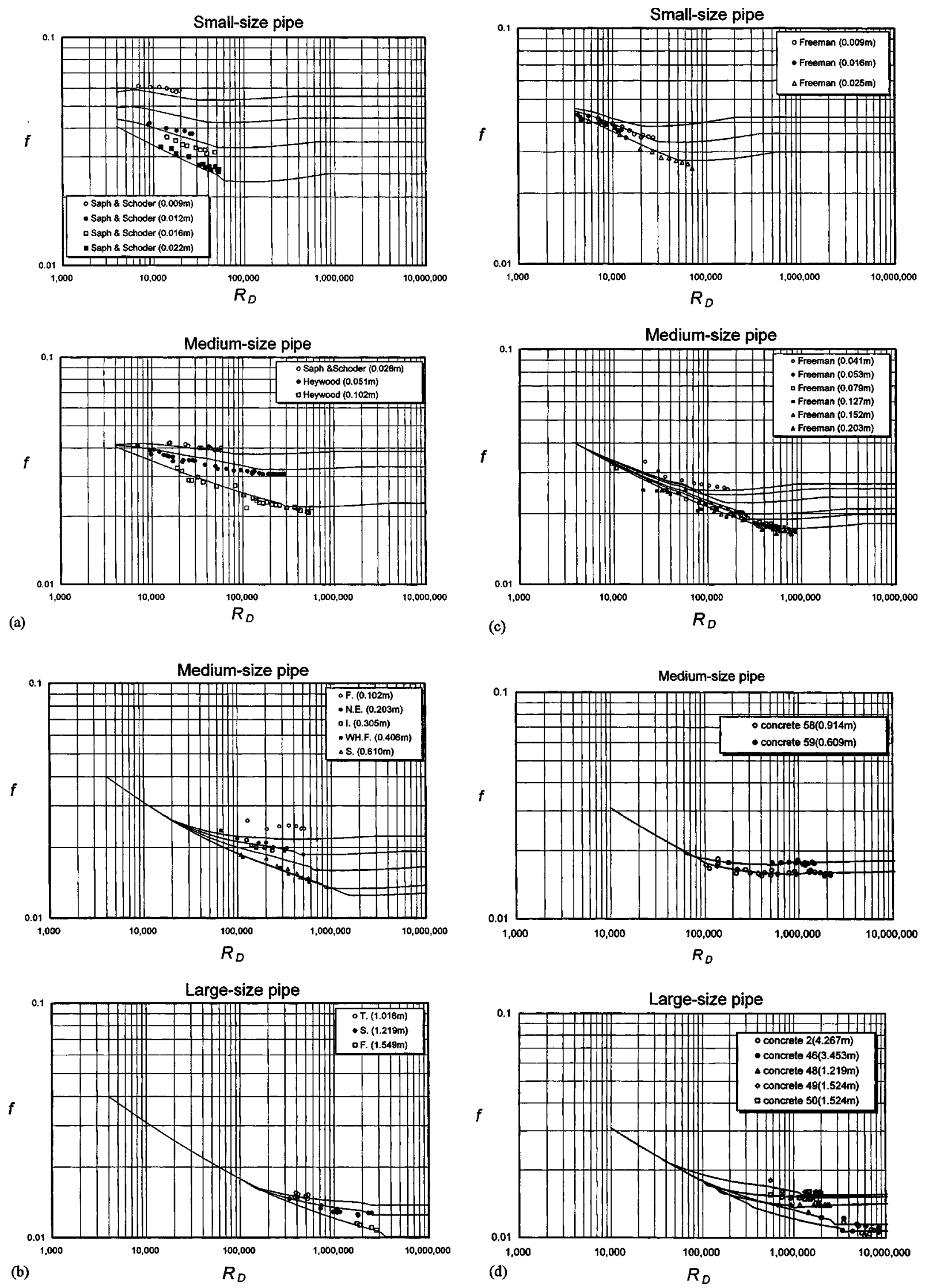

Fig. 4. Mean friction factor computed pipe friction factors plotted against measurements reported by Colebrook (1938), the U.S. Bureau of Reclamation (1965) and von Bernuth and Wilson (1989): (a) galvanized-iron pipe; (b) tar-coated cast iron pipe; (c) wrought iron pipe; (d) concrete pipe; (e) continuous interior steel pipe; (f) girth-riveted steel pipe; (g) full-riveted steel pipe; and (h) pvc pipe. 

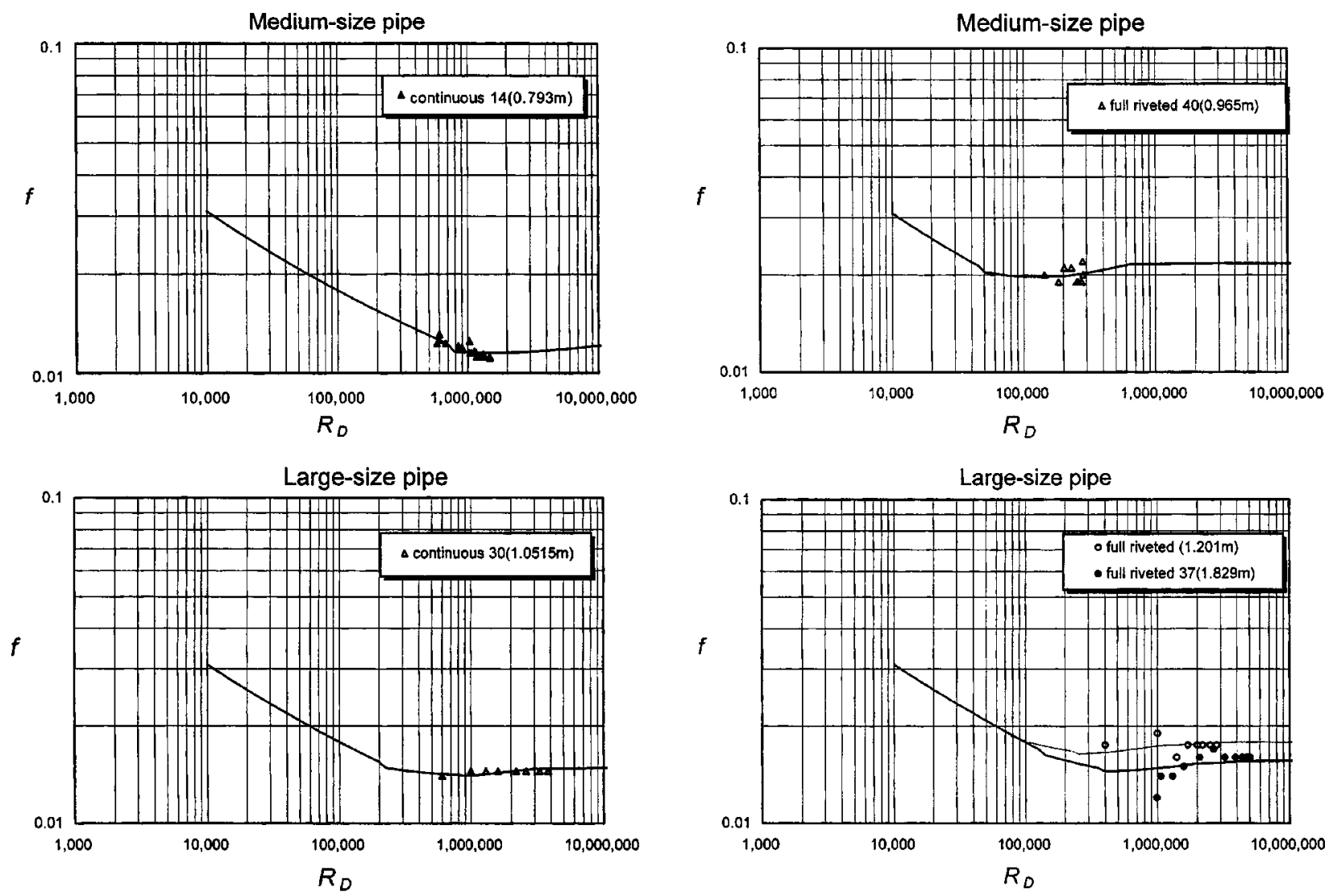

(e)

(g)
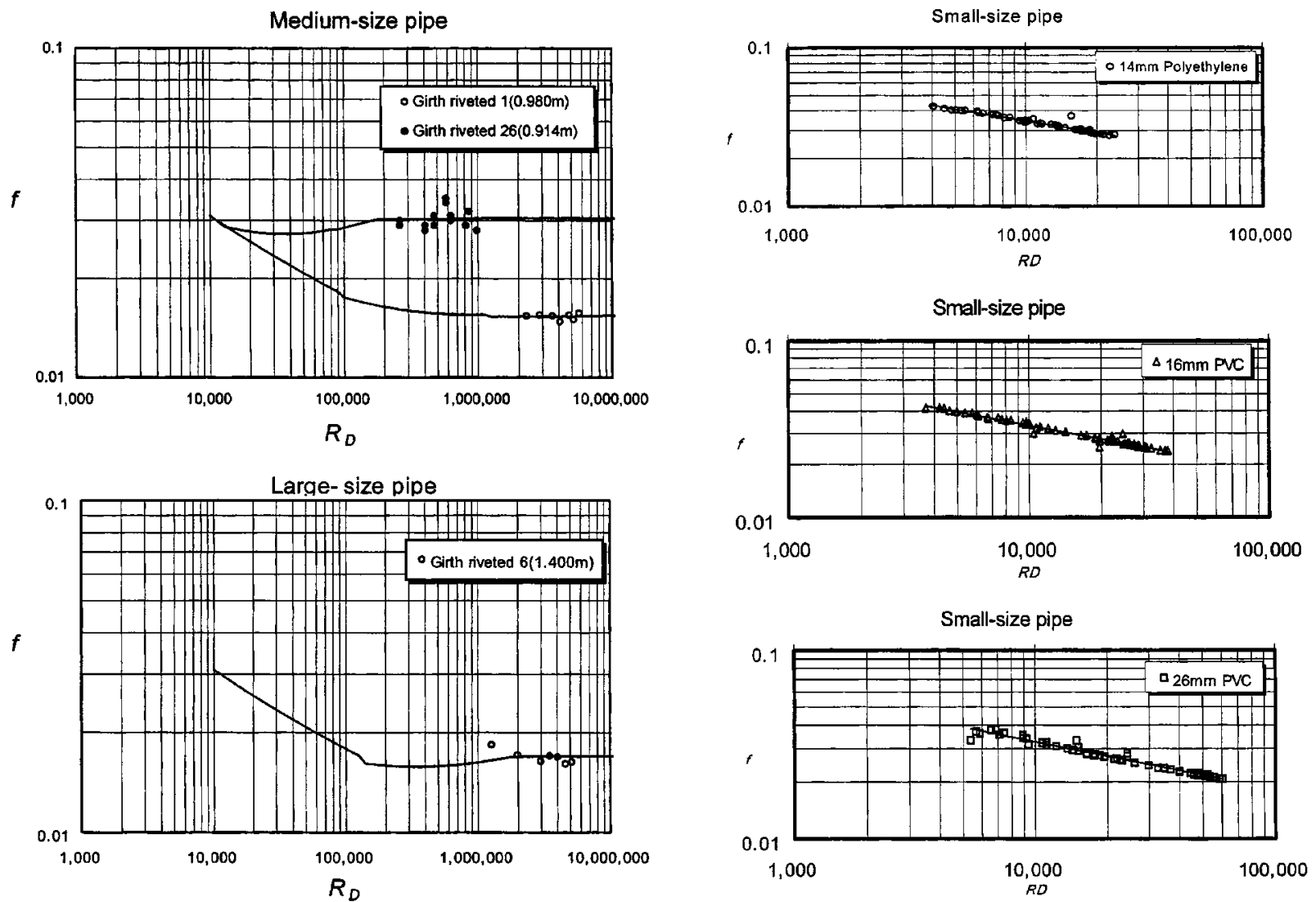

(h)

Fig. 4. (Continued). 
data for the same type of pipe, a matrix was formed by using Eq. (19) to compute the friction factor as follows:

$$
\left[\begin{array}{l}
f_{1} \\
f_{2} \\
f_{n}
\end{array}\right]=\left[\begin{array}{l}
f_{S 1} f_{R 1} \\
f_{S 2} f_{R 2} \\
f_{S n} f_{R n}
\end{array}\right]\left[\begin{array}{l}
1-P_{R} \\
P_{R}
\end{array}\right]
$$

or

$$
\left[f_{C}\right]=\left[f_{Y}\right][P]
$$

where $\left[f_{C}\right]=$ measured value of the commercial pipe friction factor; $\left[f_{Y}\right]=$ friction factor of each wall computed by adopting the roughness height with a given Reynolds number; and $[P]=$ percentage of each part. Here, $f_{C}$ and the Reynolds number are given, and both $f_{R}$ and $f_{S}$ are functions of the Reynolds number and the ratio of pipe diameter to roughness height which is unknown. By adopting different values of the roughness heights, the percentages of the rough wall were computed by the least squares method. The unknown column of percentage $[P]$ was determined as follows:

$$
[P]=\left(\left[f_{Y}\right]^{T}\left[f_{Y}\right]\right)^{-1}\left(\left[f_{Y}\right]^{T}\left[f_{C}\right]\right)
$$

where superscripts $T$ and -1 indicate the matrix transpose and the matrix inverse, respectively.

It was found from the present study that for the range of the Reynolds number considered, even the variation of the commercial pipe friction factor exhibited the same trend as that of $\mathrm{Ni}$ kuradse's laboratory results for uniformly rough pipes when the pipe component parts were not connected to each other. Fig. 3 shows the variation of friction factor of galvanized iron pipe; Fig. 3(a) for the pipe of no-connection part and Fig. 3(b) for the pipe of connection part. The solid dots indicate the measured data while the solid line indicates the values given by the ColebrookWhite equation. The roughness height of the galvanized iron pipe was estimated to be $0.03 \mathrm{~mm}$ using the laboratory data for nonconnected galvanized iron pipes. This value was used for the roughness height of the smooth part of galvanized iron pipes. Similar values were estimated for other types of commercial pipes: $0.04 \mathrm{~mm}$ for wrought iron pipes and uncoated cast iron pipes, $0.035 \mathrm{~mm}$ for tar-coated cast iron pipes and $0.02 \mathrm{~mm}$ for PVC pipes, $0.1 \mathrm{~mm}$ for concrete pipes, $0.1 \mathrm{~mm}$ for continuous interior steel pipes, $0.85 \mathrm{~mm}$ for girth riveted steel pipes, and 0.4 $\mathrm{mm}$ for fully riveted steel pipes. The Colebrook-White equation assumes that the smooth part has no roughness and the flow in the smooth part is always smooth turbulent. When the pipe diameter is relatively big this assumption might be acceptable. But when the pipe diameter is relatively small the flow in the smooth part can also be rough turbulent because the roughness ratio $D_{\mathrm{K}}$ can be small. For example, when the pipe diameter is $10 \mathrm{~mm}$, the roughness ratio of the galvanized-iron pipe becomes approximately 333. In this case, when the Reynolds number exceeds 27,200 , the flow in the smooth part surpasses the condition of smooth turbulent flow and becomes transitional turbulent flow. When the Reynolds number exceeds 380,000 , the flow in the smooth part becomes rough turbulent flow.

Using the measured data collected by Colebrook (1938) and reported by the U.S. Bureau of Reclamation (1965) and von Bernuth and Wilson (1989), the roughness heights of the rough part of a commercial pipe and the probability were estimated using regression analysis. The optimum values of the roughness heights and its probability were determined, and the computed friction factors of commercial pipes are shown in Fig. 4. The calculated

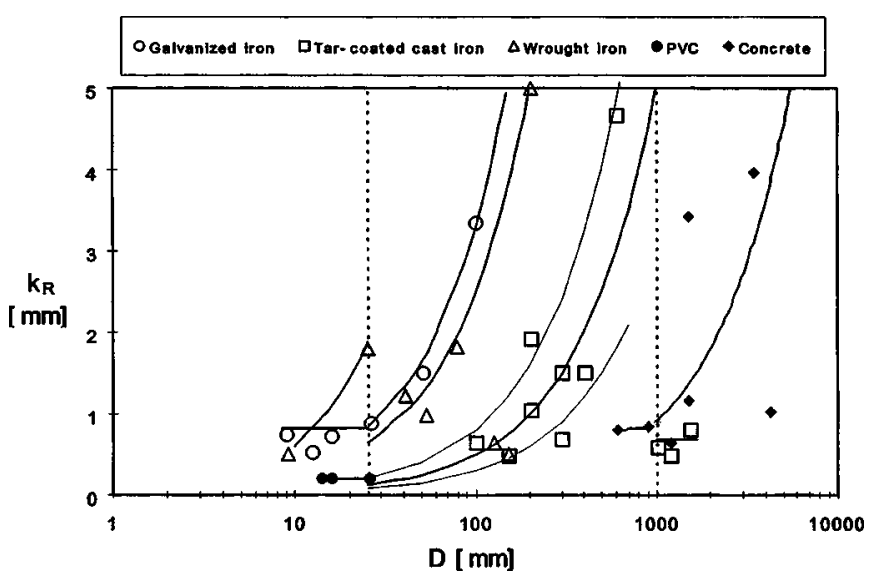

Fig. 5. Roughness height of rough wall against pipe diameter

results using MFF are in excellent agreement with the measured data for commercial pipes using the optimum values of roughness height and probability.

\section{Optimum Values of Roughness Height and Probability}

Optimum values of the roughness heights of the rough region for each pipe type were obtained empirically and for some pipes using regression analysis. These optimum values are shown in Fig. 5. Likewise, optimum values of the probability of the rough region are shown in Fig. 6. Both figures are shown with pipe diameter on the abscissa. It is recognized that while constructing these figures the amount of data used was limited, but the objective was to at least reveal a trend. Ideally one would want to have a large experimental data base but this proved unsuccessful. The roughness height of the rough part was generally found to be almost constant for small-size pipes as well as for large-size pipes, but it was found to be proportional to the diameter for medium-size pipes where it did not exceed $5 \mathrm{~mm}$. Thus, it is considered that the value of $5 \mathrm{~mm}$ may be suitable for the maximum roughness height of the medium-size pipes of $D>0.15 \mathrm{~m}$ for galvanized iron pipes, or $D>0.2 \mathrm{~m}$ for wrought iron pipes.

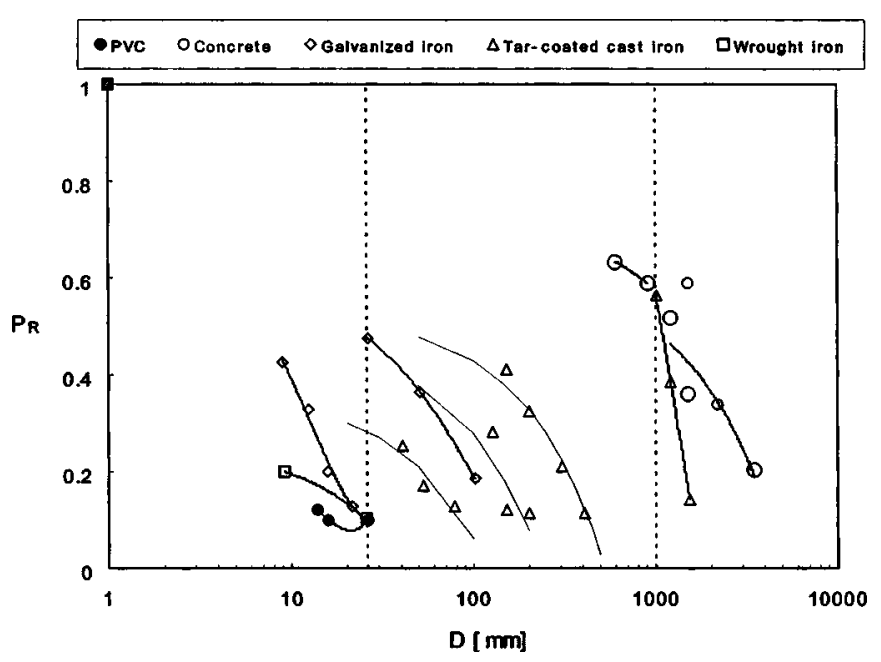

Fig. 6. Probability of rough wall against pipe diameter 
Table 1. Roughness Height of Rough Wall of Commercial Pipes

\begin{tabular}{|c|c|c|c|c|c|}
\hline Pipe type & Galvanized iron & Tar-coated cast iron & Uncoated cast-iron or Wrought iron & PVC & Concrete \\
\hline $\mathrm{k}_{\mathrm{S}}(\mathrm{mm})$ & 0.03 & 0.035 & 0.04 & 0.02 & 0.1 \\
\hline \multicolumn{6}{|l|}{$\kappa_{R}$} \\
\hline Small size $(D<25 \mathrm{~mm})$ & $0.83 \mathrm{~mm}$ & - & $0.07 D$ & $0.21 \mathrm{~mm}$ & \\
\hline \multirow[t]{3}{*}{ Medium size $\left(D<D_{m}\right)$} & $0.033 D$ & $0.008 D$ & $0.025 D$ & & \\
\hline & 2 & $0.005 D$ & & & \\
\hline & 3 & $0.003 D$ & & & \\
\hline Large size $\left(D_{m}<D\right)$ & - & $0.68 \mathrm{~mm}$ & - & & $0.0003 D+3$ or 1.0 \\
\hline
\end{tabular}

On the other hand, three regression lines of roughness height and the percentage rough part were found for the tar-coated iron pipe as shown in Figs. 5 and 6. This may be due to different types of pipe connection.

As shown in Fig. 2, Colebrook (1938) found that the roughness height was not related to the pipe diameter when the percentage of each part was not taken into account. Therefore it can be inferred that he simply took the average value of the roughness height for each type of commercial pipe. As shown in Figs. 5 and 6 , however, both roughness height and rough wall probability of commercial pipes are found related to the pipe diameter. It leads to the conclusion that the roughness of the rough part may be strongly related to the type of the pipe connection. When $D$ $<25 \mathrm{~mm}$, the pipe has a constant thickness, and when $D$ $>25 \mathrm{~mm}$ its thickness is proportional to the pipe diameter. Therefore, the roughness height of small-size pipes is almost constant irrespective of the pipe diameter, whereas the roughness height of the medium-size pipes is related to the pipe diameter. On the other hand, when the pipe diameter is bigger than $1 \mathrm{~m}$, the internal side of a connection part is normally treated manually or mechanically so that the connection part needed becomes smooth. This is considered to be the reason why the roughness height of a large-size pipe is almost constant and smaller than that of a smallsize pipe.

As noted from the results shown in Fig. 5, there is a discontinuity when the pipe diameter reaches $1 \mathrm{~m}$ for the case of a tarcoated cast iron pipe. If the pipe connection is made manually or mechanically inside the pipe so that the connection part is smooth, the proper value of the roughness height is $0.68 \mathrm{~mm}$. Otherwise, it is $5 \mathrm{~mm}$. Therefore, a commercial pipe of $1 \mathrm{~m}$ diameter can have quite different equivalent roughness heights of the rough part depending on the connection.

\section{Estimation of Roughness Height and Probability of Occupation}

The roughness heights of the rough region of a medium size pipe can be estimated as

$$
\kappa_{\mathrm{R}}=\operatorname{Min}(\alpha D, 5 \mathrm{~mm})
$$

where $\kappa_{R}=$ roughness height of the rough part of a commercial pipe. Estimated values of the parameter are given in Table 1. It is noted that $\mathrm{\kappa}_{\mathrm{R}}$ of the large tar-coated cast iron pipe is estimated to be $0.68 \mathrm{~mm}$ whereas $\kappa_{\mathrm{R}}$ of the small galvanized iron pipe is about $0.87 \mathrm{~mm}$. Some tar fluid may penetrate into the crevices at the connection part of the large tar-coated cast iron pipe, and this may reduce the roughness in the rough part.
The probability of the flow being in the rough region was also found to be related to the pipe diameter as

$$
P_{\mathrm{R}}=a+b\left(\frac{D}{D_{0}}\right)
$$

where $D_{0}=1 \mathrm{~m}$ as a standard diameter, and the coefficients $a$ and $b$ are given in Table 2. The coefficients $a$ and $b$ are constants for each pipe of the same type and the same range of diameter. The highest value of $P_{\mathrm{R}}$ was estimated to be about 0.64 . This value can be adopted as the maximum value of $P_{\mathrm{R}}$. Three regression lines of $P_{\mathrm{R}}$ were found for the wrought iron pipes as shown in Fig. 6 and Table 2.

The friction values were computed using the Colebrook-White equation and the MFF method and were compared with observed values for all the data presented by Colebrook (1938) for the galvanized iron pipe, the tar-coated cast iron pipe, and the wrought iron pipe. Comparison of the friction factor values computed by these two methods is summarized in Table 3. For economy of space, comparison of the two methods for each experimental data is omitted here. Comparing the overall average error, the MFF method gave an average error of less than $3 \%$ in the computed value of the friction factor, whereas the ColebrookWhite equation produced an average error of more than $11 \%$. This means that the use of Colebrook-White equation can over- or underestimate the pump power more than $11 \%$ from a correct value, whereas the MFF method can reduce the incorrect estimation by more than $8 \%$. However, the Colebrook-White equation gave a better estimate of the friction factor for the $1,549 \mathrm{~mm}$ diameter tar-coated cast iron pipe than did the MFF method. This was considered only an exception, and it does not mean that the MFF method is less valid for large pipes.

Table 2. Coefficients $a$ and $b$ for Estimation of $P_{R}$

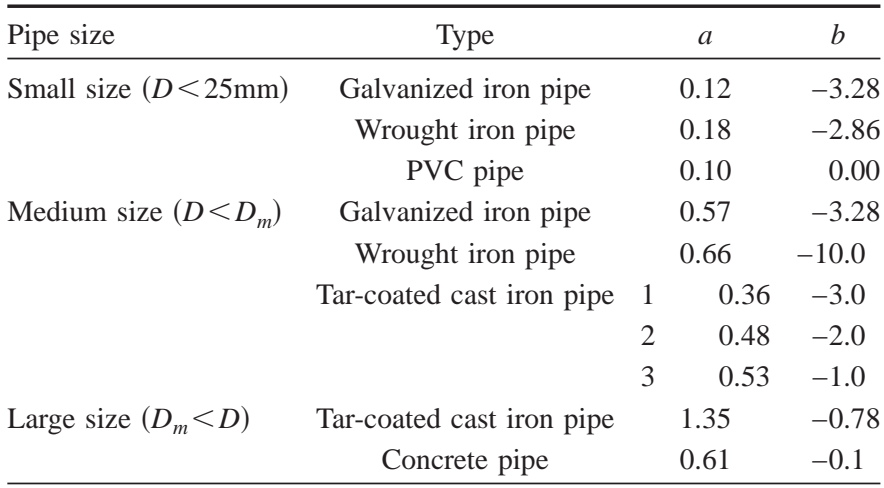


Table 3. Comparison of Error in Friction Factor Computed Using the Mean Friction Factor (MFF) Method and Colebrook-White (C-W) equation

\begin{tabular}{|c|c|c|c|c|}
\hline \multirow[b]{2}{*}{ Type of pipe } & \multirow[b]{2}{*}{ Name } & \multirow[b]{2}{*}{$D(\mathrm{~mm})$} & \multicolumn{2}{|c|}{$\begin{array}{l}\text { Average error } \\
\text { in friction factor } \\
(\%)\end{array}$} \\
\hline & & & MFF & $\mathrm{C}-\mathrm{W}$ \\
\hline \multirow[t]{7}{*}{ Galvanized iron } & S.\& S & 9 & 5.47 & 17.95 \\
\hline & S.\& S. & 12 & 7.47 & 10.36 \\
\hline & S.\& S. & 16 & 1.57 & 19.02 \\
\hline & S.\& S. & 22 & 2.16 & 28.12 \\
\hline & S.\& S. & 26 & 1.28 & 15.53 \\
\hline & H. & 51 & 1.16 & 12.32 \\
\hline & H. & 102 & 2.46 & 4.33 \\
\hline \multirow[t]{13}{*}{ Tar coated cast iron } & F. & 102 & 1.87 & 27.97 \\
\hline & N.E.(1) & 203 & 2.02 & 16.47 \\
\hline & N.E.(1) & 203 & 4.84 & 15.30 \\
\hline & I.(1) & 305 & 6.58 & 5.01 \\
\hline & F. & 305 & 2.26 & 1.96 \\
\hline & S. & 610 & 1.82 & 6.76 \\
\hline & I. $(2)$ & 305 & 3.18 & 16.77 \\
\hline & N.E. & 152 & 5.33 & 8.61 \\
\hline & F. & 203 & 4.89 & 4.62 \\
\hline & WH.F. & 406 & 1.22 & 2.13 \\
\hline & $\mathrm{T}$. & 1,016 & 1.92 & 7.13 \\
\hline & S. & 1,219 & 2.61 & 6.33 \\
\hline & F. & 1,549 & 1.19 & 0.48 \\
\hline \multirow[t]{9}{*}{ Wrought iron } & $\mathrm{F}$. & 9 & 1.10 & 4.93 \\
\hline & H.S. & 25 & 3.31 & 2.27 \\
\hline & F. & 53 & 3.08 & 40.52 \\
\hline & F. & 127 & 4.08 & 5.69 \\
\hline & F. & 203 & 2.51 & 11.29 \\
\hline & F. & 16 & 1.68 & 6.66 \\
\hline & F. & 41 & 2.63 & 15.58 \\
\hline & F. & 79 & 3.03 & 3.27 \\
\hline & F. & 152 & 2.16 & 8.49 \\
\hline Total average error & & & 2.93 & 11.24 \\
\hline
\end{tabular}

\section{Application}

The procedure is investigated using a galvanized iron pipe $10 \mathrm{~mm}$ in diameter. The flow velocity in the pipe is $1 \mathrm{~m} / \mathrm{s}$. The fluid viscosity at the existing temperature is $10^{-6} \mathrm{~m}^{2} / \mathrm{s}$. In order to compute the friction factor for a galvanized-iron pipe the following steps are involved:

1. Compute the Reynolds number: $\mathrm{R}_{D}=V D / \nu=1 \times 0.01 / 10^{-6}$ $=10,000$;

2. From Table 1, one obtains $\kappa_{S}=0.03 \mathrm{~mm}$ and $\kappa_{R}=0.83 \mathrm{~mm}$. Then, using Table 2 , the probability of the rough wall $P_{R}$ is computed as

$$
P_{R}=a+b\left(\frac{D}{D_{0}}\right)=0.12-3.28\left(\frac{0.01}{1}\right)=0.087
$$

3. The relative roughness of the rough wall against pipe diameter $D_{\kappa R}$ is computed as

$$
D_{\kappa R}=\left(\frac{D}{\kappa_{R}}\right)=\left(\frac{10}{0.83}\right)=12.05
$$

$$
\mathrm{R}_{\mathrm{\kappa} R}=\frac{V \kappa_{R}}{v}=\frac{1 \times 0.000083}{10^{-6}}=830
$$

$$
81.5<\mathrm{R}_{\mathrm{\kappa} R}<1140
$$

Therefore, the flow at the rough portion is transitional turbulent [TT]. The friction factor of the rough part $f_{R}$ is computed. Thus, one obtains from Eq. (9):

$$
\begin{gathered}
\frac{1}{\sqrt{f_{R T}}}=2 \log D_{\kappa R}+1.14, \quad f_{R T}=0.092 \\
f_{A}=0.06 D_{\kappa}^{-0.5}=0.06 \times 12.05^{-0.5}=0.0173
\end{gathered}
$$

From Eq. (13), one obtains

$$
\begin{aligned}
f_{R} & =f_{R T}-f_{A} \cos \left(0.8 \ln \mathrm{R}_{\mathrm{\kappa}}-4.06\right) \\
& =0.092-0.0173 \cos (0.8 \ln 830-4.06) \\
& =0.088
\end{aligned}
$$

4. The relative roughness of smooth wall against pipe diameter $D_{S}$ is computed:

$$
\begin{gathered}
D_{S}=\frac{D}{\kappa_{S}}=\frac{10}{0.03}=333.33 \\
\mathrm{R}_{\mathrm{\kappa} S}=\frac{V \kappa_{S}}{v}=\frac{1 \times 0.00003}{10^{-6}}=30
\end{gathered}
$$

$\mathrm{R}_{\mathrm{K} S}<81.5$. Thus the flow in the smooth wall is smooth turbulent [ST]: The friction factor of the smooth part $f_{S}$ is obtained from Eq. (8),

$$
\frac{1}{\sqrt{f_{S}}}=2 \log \mathrm{R} \sqrt{f_{S}}-0.8
$$

which by iteration yields $f_{S}=0.031$ or explicitly from, $1 / \sqrt{f_{S}}=2 \log \mathrm{R}^{0.89}-1.42 ; f_{S}=0.0308$; and

5. The mean friction factor of commercial pipes $f_{C}$ is computed as

$$
\begin{aligned}
f_{C} & =\left(1-P_{R}\right) f_{S}+P_{R} f_{R} \\
& =(1-0.087) \times 0.031+0.087 \times 0.088 \\
& =0.036
\end{aligned}
$$

which is the value sought.

\section{Summary and Conclusions}

The following concluding remarks can be made from this study: (1) empirical equations of transitional turbulent and transitional flow between laminar and rough turbulent flow have been developed, because such flow regimes may often occur in flow in commercial pipes. (2) Colebrook developed the commercial pipe friction factor equation simply by combining the zero velocity point of smooth turbulent flow and that of rough turbulent flow, assuming that both exist in the same pipe with an equal influence. He assumed that roughness is irregularly, but evenly, distributed and that the flow in the smooth and rough parts is always hydraulically smooth and rough, respectively. (3) The present study suggests two methods MZVP and MFF for calculating commercial pipe friction factors. MZVP computes the mean zero velocity 
point, and MFF directly computes the mean friction factor. Both methods consider the probability of the flow being in each region and the flow regime is determined by checking the flow condition. However, MFF is considered to be superior to MZVP, because the former can cover all flow ranges, including laminar flow. Moreover, the concept of the mean zero velocity point is somewhat contradictory to the flow conditions found in the pipes of uniform roughness. (4) In order to compute the roughness heights and percentages of occupation of commercial pipes, the least square method was employed by forming a matrix. MFF produces an almost exact agreement with measured data when using the optimum values of the roughness height and the percentage for each data set; it also produces satisfactory results when the values of the roughness height and the percentage are determined by using the relationship with the pipe diameter. The materials of the pipes tested were galvanized iron, wrought iron, cast iron, riveted steel, concrete, and PVC. (5) The roughness height and the percentage of the flow being within the rough region for various types of commercial pipes were determined using the data reported by various researchers. It is found that both values are strongly related to the pipe diameter as well as to the type of pipe. This may indicate that the roughness of the rough pipe region is strongly associated with the type of the pipe connection. (6) The present methods can be easily employed for estimating the commercial pipe friction factor, because all the equations to be used are represented by explicit forms. However, for practical use and easy manipulation of a simple design of single-lined pipes, simple forms of the power law have been suggested by the present Yoo and Singh (2004). The parameters introduced in the power law are also related with the type and size of commercial pipes.

\section{Acknowledgments}

The writers wish to express their thanks to Dr. A. Borthwick for reviewing a draft of the paper. The present study has been supported by Ajou University, which provided the financial support to equip the laboratory facility in 1995. Some of the data analysis has been conducted by Y.S. Wun, specifically for the field data collected by Colebrook (1938) and von Bernuth and Wilson (1989), and by M. I. Moon for the field data collected by the U.S. Bureau of Reclamation (1965). The majority of the work was conducted at Louisiana State University during the period of sabbatical leave of the first writer in 2002. The writers also express their gratitude to the anonymous reviewers for providing constructive reviews of the paper.

\section{Notation}

The following symbols are used in this paper:

$a=$ coefficient;

$b=$ coefficient;

$C=$ friction factor;

$D=$ pipe diameter;

$D_{0}=1 \mathrm{~m}$ as a standard diameter;

$D_{\mathrm{\kappa}}=$ relative roughness $\left(D_{\mathrm{\kappa}}=D / \kappa_{w}\right)$;

$f=$ Darcy-Weisbach friction factor $(f=8 C)$;

$f_{A}=$ friction factor amplitude $\left(f_{A}=0.06 D_{\kappa}^{-0.5}\right)$;

$f_{C}=$ mean friction factor of commercial pipes;

$f_{R}=$ friction factor of the rough part;

$f_{R T}=f$ at rough turbulent flow;

$f_{S}=$ friction factor of the smooth part;
$f_{S T}=f$ at smooth turbulent flow;

$\mathrm{R}=$ Reynolds number given by $V D / \nu$;

$\mathrm{R}_{\mathrm{K}}=$ mean velocity-roughness Reynolds number $\left(\mathrm{R}_{\mathrm{\kappa}}=V \kappa_{w} / \nu\right)$;

$\mathrm{R}_{*_{\mathrm{K}}}=$ frictional velocity-roughness Reynolds number $\left(\mathrm{R}_{*_{\kappa}}=u_{*} \mathrm{\kappa}_{w} / v\right)$;

$r=$ pipe radius;

$P_{R}=$ probability of rough wall;

$P_{S}=$ probability of smooth wall;

$u=$ local flow velocity;

$u_{*}=$ frictional velocity given by $\sqrt{\tau / \rho}$;

$V=$ cross-sectional mean velocity;

$z=$ height above a wall;

$z_{0}=$ zero velocity point at which the local velocity vanishes;

$z_{0 R}=$ zero velocity point of rough wall;

$z_{0 S}=$ zero velocity point of smooth wall;

$\kappa=$ von Karman constant $(=0.4)$;

$\kappa_{R}=$ roughness height of rough part of commercial pipe;

$\kappa_{w}=$ equivalent roughness height;

$\lambda=$ proportionality factor $\left(=3.5-0.05 \mathrm{D}_{\mathrm{K}}\right)$;

$v=$ kinetic viscosity;

$\rho=$ water density; and

$\tau=$ wall shear stress.

\section{References}

Bagarello, V., Ferro, V., Provenzano, G., and Pumo, D. (1995). "Experimental study on flow resistance law for small-diameter plastic pipes." J. Irrig. Drain. Eng., 121(5), 313-316.

Barr, D. I. H. (1976). "Discussion on technical note 128." Proc. Inst. Civ. Eng., Part 2, 61, 489-497.

Boussinesq, J. (1877). "Essay on the theory of flowing water." French Acad. Sci., 23, 1-860.

Colebrook, C. F. (1938). "Turbulent flow in pipes, with particular reference to the transition region between the smooth and rough pipe laws." J. Inst. Civ. Eng., London, 11, 133-156.

Colebrook, C. F., and White, C. M. (1937). "Experiments with fluid friction in roughened pipes." Proc. Royal Society, Series A Math. \& Phys. Sci., 161(904), 367-381.

Darcy, H. (1857). "Recherches experimentales relatives au movement de l'eaudans les tuyaux," Paris, France.

Jain, A. K., Mohan, D. M., and Khanna, P. (1978). "Modified HazenWilliams formula." J. Env. Eng. Div., ASCE, 104(EE1), 137-146.

Kamand, F. Z. (1988). "Hydraulic friction factors for pipe flow." J. Irrig. Drain. Eng., 114(2), 311-323.

Moody, L. F. (1944). "Friction factors for pipe flow." Trans. Am. Soc. Mech. Engrs., 66, 671-684.

Nikuradse, J. (1933). "Stroemungsgesetze in rauhen Rohren." Ver. Dtsch. Ing. Forsch., 361.

Prandtl, L. (1925). "Bericht ber Untersuchungen zur ausgebildeten Turbulenz." Angew. Math. Mech., 5(2), 136.

Reynolds, O. (1895). "On the dynamical theory of incompressible viscous fluids and the determination of the criterion." Philosophical Transactions, Royal Society, Series A1, 186, 123.

Singh, V. P. (1996). Kinematic wave modeling in water resources: Surface water hydrology, Wiley, New York.

Swamee, P. K., and Jain, A. K. (1976). "Explicit equations for pipe-flow problems." J. Hydraul. Div., 102(5), 657-664.

U.S. Bureau of Reclamation. (1965). "Friction factors for large conduit flowing full." Engineering Monograph, No. 7, U.S. Dept. of Interior, Washington, D.C. 
von Bernuth, R. D., and Wilson, T. (1989). "Friction factors for small diameter plastic pipes." J. Hydraul. Eng., 115(2), 183-192.

Weisbach, J. (1845). Lehrsbuch der Ingeniur und Maschinenmechanik (Textbook of Engineering Mechanics), Brunswick, Germany.

Wesseling, J., and Homma, F. (1967). "Hydraulic resistance of drain pipes." Neth. J. Agric. Sci., 15, 183-197.
Williams, G. S., and Hazen, A. (1933). Hydraulic tables, Wiley, New York.

Yen, B. (2002). “Open channel flow resistance.”J. Hydraul. Eng., 128(1), 20-37.

Yoo, D. H., and Singh, V. P. (2004). "Explicit design of commercial pipes with no secondary losses." J. Irrig. Drain. Eng., 130(5), 437-440. 